\title{
MADD promotes the survival of human lung adenocarcinoma cells by inhibiting apoptosis
}

\author{
WENXIANG BI $^{1^{*}}$, YUPING WEI ${ }^{1 *}$, JINXIANG WU ${ }^{2}$, GAOYING SUN $^{1}$, \\ YUANFANG GUO $^{1}$, QIANG ZHANG ${ }^{2}$ and LIANG DONG ${ }^{2}$ \\ ${ }^{1}$ Institute of Biochemistry and Molecular Biology, School of Medicine; ${ }^{2}$ Department of Pulmonary Medicine, \\ Qilu Hospital of Shandong University, Jinan, Shandong 250012, P.R. China
}

Received September 29, 2012; Accepted December 14, 2012

DOI: $10.3892 / o r .2013 .2258$

\begin{abstract}
MAPK-activating death domain protein (MADD) binds to the tumor necrosis factor-related apoptosis-inducing ligand (TRAIL) receptor and acts as a key downstream mediator in the TRAIL-induced apoptosis pathway. The aim of this study was to evaluate the expression of MADD in normal human and adenocarcinoma tissues of the lungs and its influence on proliferation and apoptosis of A549 human lung adenocarcinoma cells. Immunohistochemistry was carried out to detect the expression of MADD in normal and tumor tissues of the lungs. Expression of the MADD gene in A549 cells was measured by reverse transcription-polymerase chain reaction. A549 cells were transfected with plasmids carrying the DNA fragment encoding MADD and lentiviral vectors used for RNA interference, respectively. MADD expression in the transfected A549 cells was determined by western blotting. Proliferation and apoptosis were detected using MTT assay and flow cytometry, respectively. It was found that non-small cell lung cancer tissues expressed MADD at higher levels compared to normal lung tissues, and the level of MADD in lung adenocarcinoma was higher compared to that in lung squamous cell carcinoma. MADD was expressed in A549 cells. Both introduction of the DNA fragment encoding MADD and RNA interference targeting MADD effectively altered levels of MADD in the A549 cells. Overexpression of MADD in the A549 cells inhibited apoptosis and increased survival whereas abrogation of MADD promoted apoptosis and reduced cell proliferation. These results suggest that MADD may be a potential therapeutic target for lung adenocarcinoma therapy involving the TRAIL-induced apoptosis pathway.
\end{abstract}

Correspondence to: Dr Liang Dong, Department of Pulmonary Medicine, Qilu Hospital of Shandong University, 107 Wenhuaxi Road, Jinan, Shandong 250012, P.R. China

E-mail:d15506@yahoo.com.cn

*Contributed equally

Key words: MADD, TRAIL, lung cancer, immunohistochemistry, apoptosis

\section{Introduction}

The tumor necrosis factor-related apoptosis-inducing ligand (TRAIL), one member of the tumor necrosis factor (TNF) superfamily, binds to its death receptors or decoy receptors on the cell surface in the form of homotrimers and triggers TRAIL-induced apoptosis or inhibits the apoptotic pathway (1). Once TRAIL binds to its death receptors (DR4 and DR5), FAS-associated protein with death domain (FADD) and caspase- 8 or caspase- 10 are recruited to death domain motifs of the receptors, and the death-inducing signal complex (DISC) is formed, which eventually allows spontaneous cell apoptosis (2). In recent years, TRAIL and its receptors have been considered as promising targets for cancer therapy, and several molecular drugs targeting the TRAILinduced apoptosis pathway are already in clinical use (3).

The MAPK-activating death domain protein (MADD) is encoded by an mRNA formed through the post-transcriptional splicing of exons 16,21 and 26 of the insulinoma-glucagonoma clone 20 (IG20) gene (4). It is a $183-\mathrm{kDa}$ protein containing 1647 amino acids and can be phosphorylated by protein kinase B (Akt), a serine/threonine protein kinase, at three highly conserved amino acid sites: S70, T173 and T1041 (5). MADD is expressed at a low level in normal cells, a relatively higher level in the fetal brain and kidney as well as adult testis, ovarian, brain and heart tissues, and a markedly elevated level in malignant tumors of the pancreas, ovary, kidney, lung and breast $(6,7)$. After TRAIL treatment, endogenous MADD binds to the TRAIL death receptor DR4/DR5 cytoplasmic tail by its C-terminal death domain and prevents cell apoptosis by inhibiting caspase- 8 activation (8). Overexpression of MADD enhances TNF- $\alpha$-induced activation of nuclear factor- $\kappa B$ $(\mathrm{NF}-\mathrm{kB})$ and mitogen-activated protein kinase, which eventually contributes to cancer cell survival $(9,10)$. Obviously, MADD is a key downstream mediator in the TRAIL-induced apoptosis pathway and plays important roles in enhancing cell proliferation and reducing cell apoptosis.

Lung cancer, the primary malignant tumor of the lungs, is one of the leading causes of cancer-related death. It originates from the bronchial mucosa and is histologically divided into non-small cell lung cancer (NSCLC, approximately 75-80\% of lung cancer cases) and small-cell lung cancer (less than $20 \%$ of lung cancer cases). NSCLC is further divided into 
squamous cell carcinoma (SCC), adenocarcinoma (ADC) and large-cell carcinoma. This study investigated MADD expression in normal, SCC and ADC tissues of the lungs and its effects on proliferation and apoptosis of lung ADC cells. It was found that MADD was expressed at a high level and played an important anti-apoptotic role in lung ADC, which means that MADD may be a novel and effective target for therapy of lung ADC aimed at the TRAIL-induced apoptosis pathway.

\section{Materials and methods}

Immunohistochemistry (IHC). All formalin-fixed and paraffinembedded tissue samples were retrieved from the Department of Pathology of the Qilu Hospital of Shandong University. The tissue samples were procured between July 2009 and June 2011. All archival hematoxylin and eosin (H\&E)-stained slides for each patient were reviewed by two pathologists. For the use of the clinical materials for research purposes, prior patient consent and approval from the Institutional Research Ethics Committee were obtained. All of the diagnoses were made following the Pathology and Genetics of Tumors of the Lung of the World Health Organization Classification of Tumors. Clinicopathological classification and staging were determined according to the American Joint Committee on Cancer criteria.

Paraffin sections were baked at $60^{\circ} \mathrm{C}$ for $30 \mathrm{~min}$, de-paraffinized in xylene, and hydrated in descending concentrations of alcohol. Endogenous peroxidase activity was blocked with $0.3 \%(\mathrm{v} / \mathrm{v})$ hydrogen peroxide in methanol for $30 \mathrm{~min}$. Antigen retrieval was carried out by heating in a microwave oven with $0.01 \mathrm{M}$ citrate buffer, $\mathrm{pH} 6.0$, for $10 \mathrm{~min}$. The sections were then incubated with 5\% bovine serum albumin for $30 \mathrm{~min}$ at room temperature and subsequently with rabbit anti-MADD primary antibody (provided by Dr B.S. Prabhakar and Dr L.C. Li of the Department of Neurology at Illinois University School of Medicine) at $4^{\circ} \mathrm{C}$ overnight in a humidified container. Equal volumes of phosphate-buffered saline (PBS, pH 7.4) and rabbit serum were added instead of the primary antibody as blank and negative controls, respectively. The sections were then incubated with horseradish peroxidase (HRP)-conjugated secondary antibody (Beijing Zhongshan Golden Bridge Biological Technology Co., Ltd., Beijing, China) at $37^{\circ} \mathrm{C}$ for $30 \mathrm{~min}$ and stained using a DAB detection kit (Beijing Zhongshan Golden Bridge Biological Technology Co., Ltd.) followed by hematoxylin counterstain and differentiation in $\mathrm{HCl}$-alcohol. After dehydration, the gum mounted sections were photographed under an inverted microscope (BX51 TRF, Olympus, Japan). The integrated optic density (IOD) of the positive area in each microphotograph was assessed using immunohistochemical analysis software (Image-Pro Plus 6.0).

Cell culture. The human lung adenocarcinoma A549 cell line and the human embryonic kidney (HEK) 293T cell line were purchased from the China Center for Type Culture Collection (CCTCC, Shanghai, China). The A549 and HEK293T cells were separately cultured in Ham's F12 medium (Thermo Fisher Scientific Inc., Rockford, IL, USA) and DMEM (high glucose) (Hyclone, Logan, UT, USA), both of which contained $10 \%$ fetal bovine serum (FBS, Hyclone), $100 \mathrm{U} / \mathrm{ml}$ penicillin and $100 \mu \mathrm{g} / \mathrm{ml}$ streptomycin, at $37^{\circ} \mathrm{C}$ in a humidified atmo- sphere with 5\% $\mathrm{CO}_{2}$. At $80-90 \%$ confluence, the cells were subcultured at a ratio of $1: 3$ with $0.25 \%$ trypsin.

$R N A$ extraction and reverse transcription-polymerase chain reaction (RT-PCR). Total RNA was extracted from A549 cells using TRIzol reagent (DBI, Biosciences, USA). Reverse transcription was carried out with $1 \mu \mathrm{g}$ of total RNA using a ReverTra Ace qPCR RT kit (Toyobo, Osaka, Japan). Briefly, RNA and random primers in a $12-\mu 1$ reaction mixture were put on ice immediately after incubation at $65^{\circ} \mathrm{C}$ for $5 \mathrm{~min}$. Then, cDNAs were synthesized in a $20-\mu 1$ reaction mixture containing ReverTra Ace, dNTP mix, and RNase inhibitor at $30^{\circ} \mathrm{C}$ for $10 \mathrm{~min}$ followed by $42^{\circ} \mathrm{C}$ for $60 \mathrm{~min}, 90^{\circ} \mathrm{C}$ for $5 \mathrm{~min}$, and $4^{\circ} \mathrm{C}$ for $5 \mathrm{~min}$. For PCR amplification, $2 \mu \mathrm{l}$ of cDNA was added to a $50-\mu 1$ reaction mixture containing $25 \mu \mathrm{l} 2 \mathrm{X}$ Taq PCR Master mix (DBI, Bioscience, USA) and $0.01 \mu \mathrm{M}$ of each primer. The reaction mixture was first incubated at $50^{\circ} \mathrm{C}$ for $30 \mathrm{~min}$ and $94^{\circ} \mathrm{C}$ for $2 \mathrm{~min}$. Subsequently, 30 cycles of PCR (denaturation at $94^{\circ} \mathrm{C}$ for $30 \mathrm{sec}$, annealing at $55^{\circ} \mathrm{C}$ for $30 \mathrm{sec}$, and extension at $72^{\circ} \mathrm{C}$ for $1 \mathrm{~min}$ ) were carried out with a final incubation at $72^{\circ} \mathrm{C}$ for $7 \mathrm{~min}$. Two primer sets, F1/R1 (5'-CGG GACTCTGACTCCGAACCTAC-3' and 5'-GCGGTTCAG CTTGCTCAGGAC-3') and F2/R2 (5'-CTGCAGGTGACCC TGGAAGGGATC-3' and 5'-TGTACCCGGGTCAGCTAG AGACAGGCC-3') were separately used to amplify the desired fragments of the IG20 gene as previously reported (4). The primers $(\mathrm{F} / \mathrm{R})$ for glyceraldehyde-3-phosphate dehydrogenase (GAPDH) were provided in the ReverTra Ace qPCR RT kit. PCR products were separated on a 5\% polyacrylamide gel. The gel was stained with ethidium bromide and photographed under a UV transilluminator (Alpha Imager ${ }^{\mathrm{TM}}$ 2200, San Leandro, CA, USA).

Plasmid transfection. A549 cells were seeded into 6-well plates at a density of $2 \times 10^{5}$ cells/well and cultured in $2 \mathrm{ml}$ Ham's F-12 medium containing 10\% FBS without antibiotics for $24 \mathrm{~h}$ in a $\mathrm{CO}_{2}$ incubator. Lipofectamine $2000(10 \mu \mathrm{l})$ (Invitrogen, Carlsbad, CA, USA) and $4 \mu \mathrm{g}$ pEYFP or pEYFP-MADD plasmid DNA (provided by Prabhakar and Li as previously noted) were separately diluted in $250 \mu$ l portions of Opti-MEM reduced serum medium (Invitrogen). After $5 \mathrm{~min}$ of incubation at room temperature, the diluted Lipofectamine 2000 and plasmid DNA were mixed together. The DNA-liposome complex was incubated for $20 \mathrm{~min}$ at room temperature and then directly added to the cultured cells in $2 \mathrm{ml}$ of Opti-MEM medium. The culture medium was replaced with Ham's F-12 medium containing 10\% FBS without antibiotics after transfection for $6 \mathrm{~h}$ in the $\mathrm{CO}_{2}$ incubator. Expression of MADD in the transfected cells was detected using western blot assay after $48 \mathrm{~h}$ of incubation.

Lentiviral vector transfection. HEK293T cells were seeded in $100-\mathrm{mm}$ plates at a density of $1.5 \times 10^{6}$ cells/plate and cultured for $24 \mathrm{~h}$. Lentiviruses were produced by co-transfecting $16 \mu \mathrm{g} \mathrm{pNL}-\mathrm{SIN}-\mathrm{GFP}-\mathrm{SCR}$ or pNL-SIN-GFP-MID lentiviral vectors (provided by Prabhakar and $\mathrm{Li}$ as previously noted) and $32 \mu 1$ Lenti-Pac HIV Mix (GeneCopoeia, Rockville, MD, USA) into HEK293T cells. The medium was replaced with fresh medium after transfection for $16 \mathrm{~h}$. The lentiviruscontaining supernatant was harvested after incubation for $40 \mathrm{~h}$ 
and filtered using a $0.45-\mu \mathrm{m}$ filter. The optimal viral titer was determined by transfecting HEK293T cells using a previously described method (11). A549 cells were seeded in 6-well plates at a density of $5 \times 10^{5}$ cells/well and cultured in 2-ml complete medium. After incubation for $24 \mathrm{~h}$, the medium was replaced with serum-free medium containing $8 \mu \mathrm{g} / \mathrm{ml}$ Polybrene (Sigma, St. Louis, MO, USA), and the viral supernatant was added to the cells in multiples of infection of 10 . After $6 \mathrm{~h}$ of incubation for transfection, the medium was replaced with complete medium, the cells were incubated for a further $48 \mathrm{~h}$, and expression of MADD in the transfected cells was detected using western blot assay.

Protein determination and western blot analysis. The transfected cells were lysed with RIPA lysis buffer (Thermo Fisher Scientific Inc.). After centrifugation at $13,000 \mathrm{x}$ f for $20 \mathrm{~min}$ at $4^{\circ} \mathrm{C}$, protein concentrations in the cell lysates were determined using a BCA protein assay kit (Beyotime, Jiangsu, China). Samples corresponding to $20 \mu \mathrm{g}$ of protein were fractionated by $8 \%$ sodium dodecyl sulfate polyacrylamide gel electrophoresis and then transferred to a polyvinylidene difluoride membrane. The membrane was blocked with TBST buffer (20 mM Tris-buffered saline with $0.1 \%$ Tween-20) containing $5 \%$ non-fat dry milk for $1 \mathrm{~h}$ at room temperature and incubated with primary antibodies targeting $\beta$-actin (Beijing Zhongshan Golden Bridge Biological Technology Co., Ltd.) and MADD at $4^{\circ} \mathrm{C}$ overnight. The next day, the membrane was washed 3 times for 5 min each time with TBST buffer and incubated with HRP-conjugated secondary antibody at room temperature for $1 \mathrm{~h}$. After the membrane was washed once with TBS (20 mM Tris-buffered saline) for $15 \mathrm{~min}$ and twice with TBST for $5 \mathrm{~min}$ each at room temperature, MADD and $\beta$-actin on the membrane were detected using Pierce ECL Western blotting substrate (Thermo Fisher Scientific Inc.) by a chemiluminescence apparatus (FluorChem $\hat{A}^{\circledR} \mathrm{Q}$, Cell Biosciences, Santa Clara, CA, USA).

MTT assay. A549 cells were seeded into 96-well plates at a density of $5 \times 10^{3}$ cells/well (for plasmid transfection) or $1 \times 10^{4}$ cells/well (for lentiviral vector transfection) and incubated for $24 \mathrm{~h}$. The A549 cells were respectively transfected with pEYFP and pEYFP-MADD plasmids as well as pNL-SIN-GFP-SCR and pNL-SIN-GFP-MID lentiviral vectors using the methods as described above. After transfection for $48 \mathrm{~h}, 10 \mu \mathrm{l}$ of $5 \mathrm{mg} / \mathrm{ml}$ 3-(4,5-dimethylthiazol-2-y1)-2,5-diphenytetrazolium bromide (MTT, Sigma) was added to each well, and the cultures were incubated for an additional $4 \mathrm{~h}$. Then formazan crystals were dissolved by the addition of dimethyl sulfoxide (DMSO, Sigma) solution. The absorbance of each well was measured at $490 \mathrm{~nm}$ (Model 680, Bio-Rad Laboratories, Hercules, CA, USA).

Flow cytometric (FCM) analysis. Cell apoptosis was detected by flow cytometry using an Annexin V-FITC/PI apoptosis detection kit (KeyGen, China). A549 cells were seeded into a 6 -well plate at $2 \times 10^{5}$ or $4 \times 10^{5}$ cells/well and transfected with pEYFP-MADD plasmids or pNL-SIN-GFP-MID lentiviral vectors as described in the Plasmid transfection and Lentiviral vector transfection sections. After incubation for $48 \mathrm{~h}$, the transfected cells were collected, washed twice with ice-cold
Table I. Clinicopathological characteristics of the tumor tissues.

Tumor characteristics

No. of cases

$\begin{array}{ll}\text { Histology } & \\ \text { SCC } & 31 \\ \text { ADC } & 17\end{array}$

Degree of differentiation

High

Moderate 20

Low 21

Stage

I 14

II 25

III 9

Table II. IOD of the IHC images.

\begin{tabular}{lcc}
\hline Tissues & No. of cases & IOD $($ means \pm SD) \\
\hline Normal & 20 & $16.419 \pm 0.606$ \\
SCC & 31 & $26.358 \pm 0.759^{\mathrm{a}}$ \\
ADC & 17 & $30.065 \pm 0.962^{\mathrm{a}, \mathrm{b}}$ \\
\hline
\end{tabular}

${ }^{\mathrm{a}} \mathrm{P}<0.01$ vs. normal lung tissues. ${ }^{\mathrm{b}} \mathrm{P}<0.01$ vs. $\mathrm{SCC}$. IOD, integrated optical density.

PBS, and resuspended in 500- $\mu 1$ binding buffer. Annexin V/ FITC $(5 \mu \mathrm{l})$ and $5 \mu \mathrm{l}$ PI were sequentially added to the cell suspension. The cell suspension was incubated at room temperature in the dark for $15 \mathrm{~min}$, and the cells were immediately analyzed on a FACS flow cytometer (Becton-Dickinson, Franklin Lakes, NJ, USA). Annexin V/FITC-positive and PI-negative cells were scored as apoptotic.

Statistical analysis. All of the experiments were conducted at least three times. Data are shown as the means \pm SD. Statistical significance was determined by statistical analysis software SPSS 17.0. $\mathrm{P}<0.05$ was considered to indicate a statistically significant result.

\section{Results}

Expression of MADD in normal lung tissues and NSCLC. In this study, the clinicopathological features of 68 samples of tissues of the lungs were analyzed. These included 20 samples of normal tissues, 31 samples of SCC tissues and 17 samples of ADC tissues. There was no significant difference in gender and age among the three groups. Clinicopathological characteristics of the tumor tissues are summarized in Table I. IHC results are shown in Fig. 1 and Table II. MADD was expressed in both normal lung and NSCLC tissues. IOD analysis revealed that the expression level of MADD in ADC or SCC was higher than that in the normal lung tissues $(\mathrm{P}<0.01)$. The mean IOD values of the ADC and SCC tissues were 1.95 and 

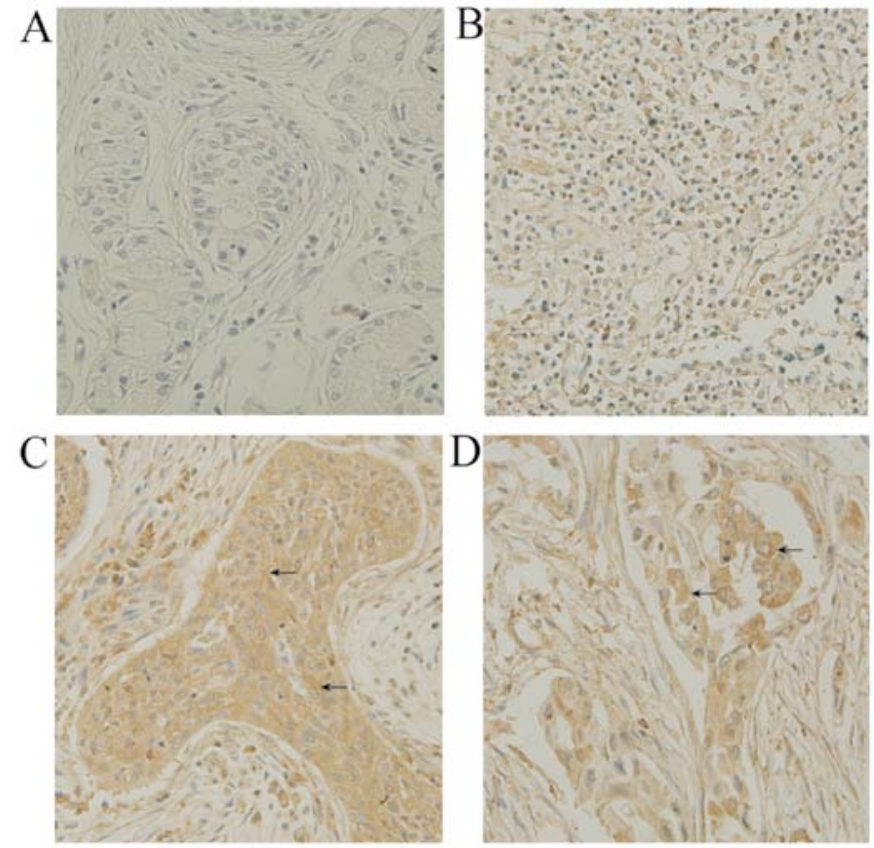

Figure 1. Representative images of immunohistochemical staining of MADD in normal lung tissues (A and B) as well as lung SCC (C) and ADC (D) tissues captured at a magnification of $x 40$. (A) Blank control. Black arrows indicate brown cytoplasmic staining of the MADD protein.

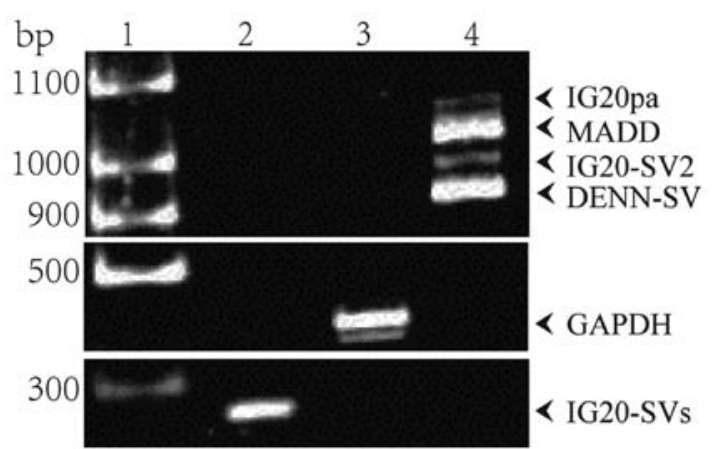

Figure 2. Expression of the IG20 gene at the transcriptional level in A549 cells. Lane 1, marker; lane 2, RT-PCR products amplified with F2/R2 primers; lane 3, RT-PCR products amplified with F/R primers; lane 4, RT-PCR products amplified with F1/R1 primers.

1.61 times that of normal lung tissues, respectively. It was also found that ADC expressed more MADD than SCC $(\mathrm{P}<0.01)$. The mean IOD of ADC tissues was 1.22 times that of SCC tissues. In addition, IOD analysis showed that levels of MADD expression in both the ADC and SCC tissues were not related to differentiation and stage of ADC and SCC.

Expression of the IG20 gene in A549 cells. As MADD was expressed at a relatively higher level in ADC, all subsequent studies were carried out using the human lung adenocarcinoma cell line A549. First, RT-PCR assay was applied to examine mRNA expression of the IG20 gene in A549 cells since MADD is a splice variant of IG20. RT-PCR results are shown in Fig. 2. Four spliced transcripts, $\sim 1 \mathrm{~kb}$ in length, were amplified with the primer pair F1 and R1 that targets exons $13 \mathrm{~L}$ and 16 of the IG20 gene (Fig. 2, lane 4). These
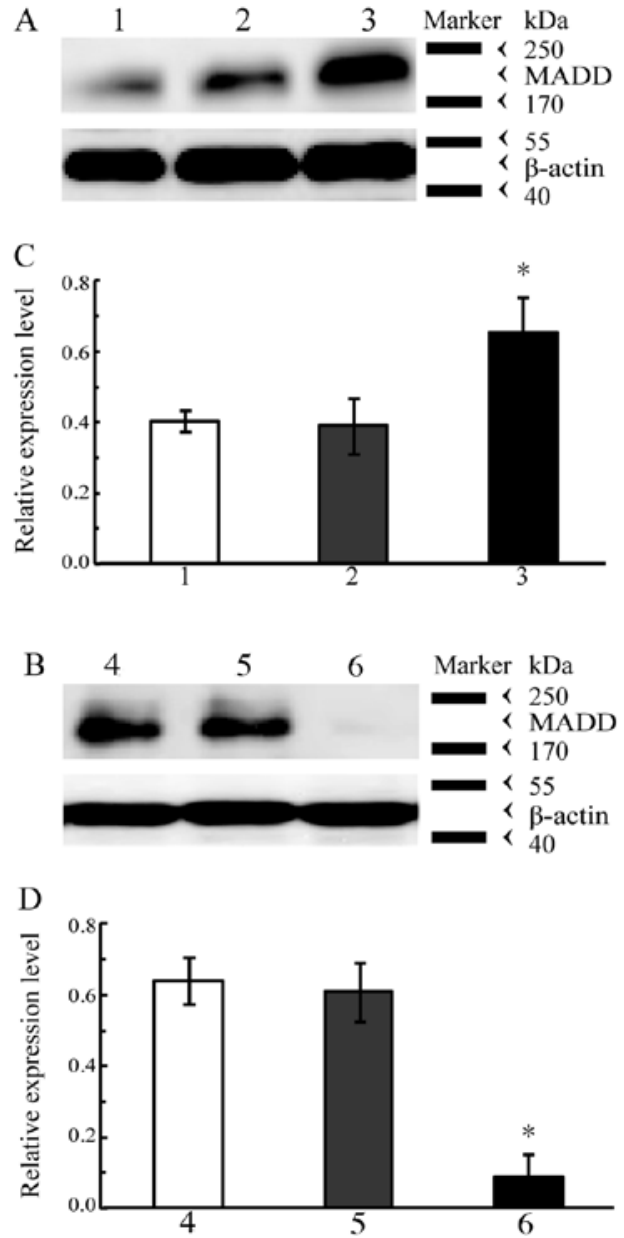

Figure 3. Western blot analyses of MADD expression in A549 cells. (A) A549 cells were transfected with pEYFP or pEYFP-MADD plasmids. Lane 1, control; lane 2, transfection with pEYFP; lane 3, transfection with pEYFP-MADD. (B) A549 cells were transfected with pNL-SIN-GFP-SCR or pNL-SIN-GFP-MID lentiviral vectors. Lane 4, control; lane 5, transfection with pNL-SIN-GFP-SCR; lane 6, transfection with pNL-SIN-GFP-MID. Quantitative analysis of the results is also shown. (C) Quantitative analysis of MADD expression in A549 cells transfected with pEYFP or pEYFP-MADD plasmids. (D) Quantitative analysis of MADD expression in A549 cells transfected with pNL-SIN-GFP-SCR or pNL-SIN-GFP-MID lentiviral vectors. Data are means \pm SD from three independent experiments $\left({ }^{*} \mathrm{P}<0.01\right.$ vs. control).

are the transcripts encoding IG20pa, MADD, IG20-SV2 and DENN-SV. With the primer pair F2 and R2 targeting exon 34 of the IG20 gene, only one fragment of $\sim 300 \mathrm{bp}$ was amplified (Fig. 2, lane 2), which means that the transcripts encoding KIAA0358 and IG20-SV4 were undetectable in A549 cells. These results indicated that the four splice variants of IG20, which included MADD, were expressed in A549 cells.

MADD expression in transfected A549 cells. In order to assess the function of MADD in A549 cells, we introduced the exogenous MADD gene into the cells through transfection and silencing of MADD expression using RNA interference (RNAi). The expression of MADD in the transfected A549 cells was determined by western blot analysis. The results of western blot analysis are shown in Fig. 3. The level of MADD obviously increased in the A549 cells containing the exogenous MADD gene $(\mathrm{P}<0.01$ vs. control, Fig. $3 \mathrm{~A}$ and $\mathrm{C})$ 

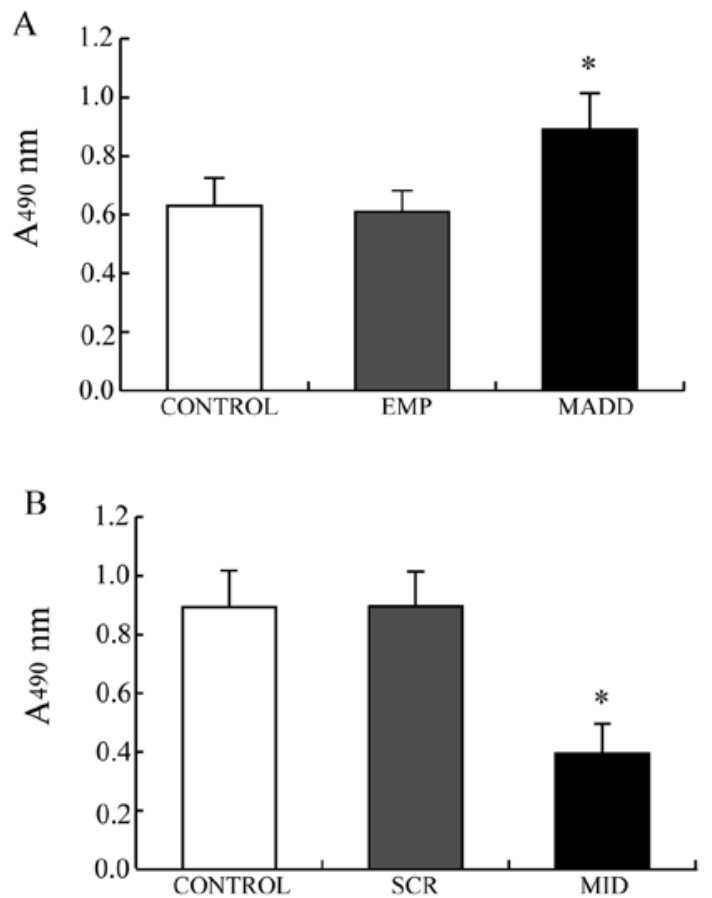

Figure 4. Results of the MTT assay. (A) A549 cells were transfected with the pEYFP (EMP) or pEYFP-MADD (MADD) plasmids. (B) A549 cells were transfected with the pNL-SIN-GFP-SCR (SCR) or pNL-SIN-GFP-MID (MID) lentiviral vectors. Data represent the means $\pm \mathrm{SD}$ of triplicate samples ( $\mathrm{P}<0.01$ vs. control)

Table III. Apoptosis rate of A549 cells, $n=3$.

\begin{tabular}{lc}
\hline Group & Apoptosis rate $(\%)($ means \pm SD) \\
\hline Control & $2.4 \pm 0.5$ \\
Overexpressing MADD & $1.4 \pm 0.6^{\mathrm{a}}$ \\
Silencing MADD & $6.7 \pm 1.5^{\mathrm{a}}$
\end{tabular}

${ }^{\mathrm{a}} \mathrm{P}<0.01$ vs. control.

whereas it was markedly decreased in the A549 cells subjected to RNAi targeting of MADD ( $\mathrm{P}<0.01$ vs. control, Fig. 3B and D); both introduction of the exogenous MADD gene and RNAi targeting of MADD effectively altered the MADD expression levels in A549 cells.

Effects of MADD on A549 cell proliferation. MTT assay was performed to observe the effects of MADD on the proliferation of A549 cells. As showed in Fig. 4, the A549 cells transfected with the pEYFP-MADD plasmids, in which MADD was overexpressed, demonstrated greater viability than the control cells $(\mathrm{P}<0.01$, Fig. 4A) while the viability of the A549 cells transfected with the pNL-SIN-GFP-MID lentiviral vectors, with silenced expression of MADD, was lower than that of the control cells $(\mathrm{P}<0.01$, Fig. 4B). Obviously, MADD promotes proliferation of A549 cells.

Effects of MADD on the apoptosis of A549 cells. To further investigate the mechanisms by which MADD promotes
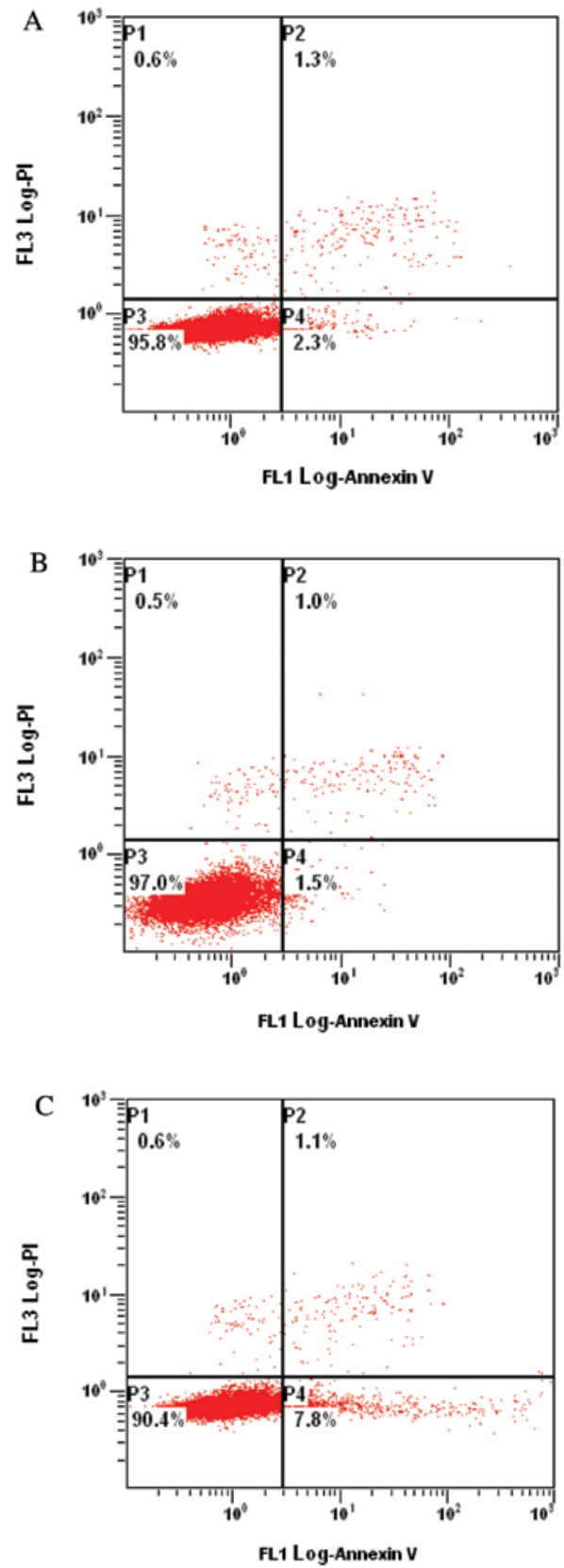

Figure 5. Representative charts of FCM detecting the apoptosis in the (A) untreated A549 cells and (B) A549 cells transfected with the pEYFPMADD plasmids and (C) pNL-SIN-GFP-MID lentiviral vectors. The results shown are dot plots from two-color flow cytometry: lower-left quadrant, viable; upper-left quadrant, necrotic; lower-right quadrant, early apoptotic; upper-right quadrant, late apoptotic cells.

proliferation of A549 cells, FCM was utilized to evaluate the apoptosis of A549 cells. FCM results are shown in Table III and Fig. 5. The apoptosis rate of control cells was $2.4 \pm 0.5 \%$. The apoptosis rate of the A549 cells transfected with the pEYFPMADD plasmids decreased to $1.4 \pm 0.6 \%$, whereas that of the A549 cells transfected with the pNL-SIN-GFP-MID lentiviral vectors increased to $6.7 \pm 1.5 \%$. These results demonstrated that overexpression of MADD effectively inhibits A549 cell apoptosis, and down-modulation of MADD obviously enhances apoptosis, which suggests that in A549 cells, MADD distinctly affects apoptosis and functions as an inhibitor of apoptosis. 


\section{Discussion}

The worldwide incidence of lung cancer has rapidly increased in recent years. The exact pathogenesis of lung cancer is still unclear. Studies have revealed that the development of lung cancer is closely associated with abnormal alterations in oncogenes, tumor-suppressor genes, cytokines and their receptors and cell adhesion molecules (12-14). For early-stage lung cancer without overt metastasis, surgery is the optimal choice while it has great limitations for advanced-stage or metastatic lung cancer. However, the fact is that most patients with lung cancer are diagnosed with advanced-stage disease or metastasis. Although radiotherapy and chemotherapy are also feasible therapies for lung cancer, severe side effects and drug tolerance hamper their application. Thus, it is of vital practical significance to explore new therapeutic targets and approaches for lung cancer.

TRAIL is a promising candidate for cancer therapy as it can selectively induce apoptosis in tumor cells and effectively kill them but not normal cells (15). Interaction between TRAIL and its cell surface receptors DR4/DR5 can result in intracellular recruitment of FADD and caspase-8/10 and formation of DISC, which can eventually trigger apoptosis of tumor cells (2). DR1 and DR2, two receptors of TRAIL that are only expressed on normal cell membranes, are considered as decoy receptors since they have no or only part of a death domain. As a result, after binding to TRAIL, DR1 and DR2 fail to transmit apoptotic signals and hardly induce apoptosis in normal cells $(16,17)$. At present, some molecular antitumor drugs targeting the TRAIL-induced apoptosis pathway have been developed, which can kill tumor cells without obvious toxicity and side effects to normal cells (18). However, tumor cells can easily acquire resistance to TRAIL therapy (19). Overexpression of decoy receptors and invalid mutations of TRAIL receptors together with loss of caspase- 8 expression due to CASP gene methylation can cause TRAIL resistance in tumor cells $(20,21)$. Additionally, NF- $\mathrm{\kappa B}$ is always activated downstream in the TRAIL pathway and may more easily cause tumor cell metastasis (22). Hence, TRAIL strategy for cancer therapy needs further improvement.

The IG20 gene was first discovered in $\beta$ cells of human pancreas islets by Cunningham in 1996 (23). It is located on human chromosome 11p11, having 5995 bp and containing 36 exons $(6,24)$. By alternative splicing of exons 13L, 16, 21, 26 and 34, it can encode at least six protein isoforms: KIAA0358, IG20-SV4, IG20pa, MADD, DENN and IG20-SV2 (4). KIAA0358 and IG20-SV4 are mainly expressed in the central nervous system such as the cerebral cortex, hippocampus and spinal cord and are involved in neurotransmission (25-28) and neuro-degeneration (29). IG20pa, IG20-SV2, MADD and DENN-SV are expressed in almost all normal human tissues at low levels and participate in the TRAIL-mediated signaling pathway (4). IG20pa and IG20-SV2 have no tissue and cell specificity. MADD and DENN-SV proteins are expressed at higher levels compared to other protein isoforms (30) and are obviously increased in tumor cells and tissues (30-33), which suggests that they play important roles not only in survival of normal cells but in pathogenesis and development of tumors.

According to previous studies, continuous expression of MADD can render cells more resistant to TRAIL-induced apoptosis (30). Phosphorylated MADD can specifically interact with TRAIL receptors to suppress cell apoptosis by means of inhibiting the cleavage of procaspase- 8 (8). Antisense abrogation of MADD expression makes tumor cells more susceptible to extracellular apoptotic stimuli such as TNF- $\alpha$, TRAIL, etoposide and vinblastine (24) as well as $\gamma$-irradiation (34). MADD expression of tumor cells markedly differs from that of normal cells (4). Therefore, studies of the functions of MADD and regulatory mechanisms of its expression will help find novel and effective targets for TRAIL therapy against tumors.

To date, the roles of MADD in lung cancer have not been elucidated. This study mainly investigated expression of MADD in lung ADC tissues and its function in lung ADC cells. IHC analysis was used to observe MADD expression in normal and NSCLC tissues of the lungs. The results of IHC revealed that SCC and ADC tissues expressed MADD at a higher level when compared with normal lung tissues, which implies that MADD may play an important role in the pathogenesis of NSCLC. It was also found that ADC tissues had a higher MADD level than SCC tissues, indicating that ADC and SCC have different pathogenic mechanisms, and MADD may play a more vital role in ADC development. Hence, we used human lung adenocarcinoma A549 cells to further investigate the function of MADD in lung cancer. RT-PCR results showed that A549 cells expressed MADD, thus A549 cells were transfected with pEYFP-MADD plasmids or pNL-SIN-GFP-MID lentiviral vectors to increase or decrease MADD expression. MADD expression in the transfected A549 cells and cell proliferation were detected by means of western blot analysis and MTT assay, respectively. Results demonstrated that A549 cells overexpressing MADD exhibited an increased survival rate whereas silencing of MADD expression inhibited A549 cell viability. Since MADD has no obvious effect on the cell cycle (35), we applied FCM to access the influence of MADD on A549 cell apoptosis and found that MADD obviously affected the apoptosis of A549 cells. The apoptosis rate of MADD-overexpressing A549 cells was only $58.3 \%$ of that of the control cells, whereas the apoptosis rate of the MADD-silenced A549 cells was 2.8 times that of control cells, indicating that MADD strongly inhibits the apoptosis of A549 cells. These results elucidated the function of MADD in lung ADC and provides an important foundation and a promising future for improving the effects of TRAIL therapy on lung ADC by regulation and control of MADD expression and activity.

\section{Acknowledgements}

We would like to express our sincere appreciation and gratitude to Dr B.S. Prabhakar and Dr L.C. Li of the Department of Neurology at Illinois University School of Medicine for their generosity in providing us with the primary antibody against MADD and the pEYFP and pEYFP-MADD plasmids as well as the pNL-SIN-GFP-SCR and pNL-SINGFP-MID lentiviral vectors.

\section{References}

1. Niemoeller OM and Belka C: Radiotherapy and TRAIL for cancer therapy. Cancer Lett: July 12, 2011 (Epub ahead of print). 
2. Johstone RW, Frew AJ and Smyth MJ: The TRAIL apoptotic pathway in cancer onset, progression and therapy. Nat Rev Cancer 10: 782-798, 2008.

3. Kruyt FA: TRAIL and cancer therapy. Cancer Lett 263: 14-25, 2008.

4. Al-Zoubi AM, Efimova EV, Kaithamana S, Martinez O, El-Idrissi Mel-ADogan RE and Prabhakar BS: Contrasting effects of IG20 and its splice isoforms, MADD and DENN-SV, on tumor necrosis factor alpha-induced apoptosis and activation of caspase-8 and -3. J Biol Chem 276: 47202-47211, 2001.

5. Li P, Jayarama S, Ganesh L, et al: Akt-phosphorylated mitogenactivated kinase-activating death domain protein (MADD) inhibits TRAIL-induced apoptosis by blocking Fas-associated death domain (FADD) association with death receptor 4. J Biol Chem 285: 22713-22722, 2010.

6. Chow VT, Lim KM and Lim D: The human DENN gene: genomic organization, alternative splicing, and localization to chromosome 11p11.21-p11.22. Genome 41: 543-552, 1998.

7. Efimova EV, Al-Zoubi AM, Martinez O, et al: IG20, in contrast to DENN-SV, (MADD splice variants) suppresses tumor cell survival, and enhances their susceptibility to apoptosis and cancer drugs. Oncogene 23: 1076-1087, 2004.

8. Mulherkar N, Prasad KV and Prabhakar BS: MADD/DENN splice variant of the IG20 gene is a negative regulator of caspase- 8 activation. Knockdown enhances TRAIL-induced apoptosis of cancer cells. J Biol Chem 282: 11715-11721, 2007.

9. Mulherkar N, Ramaswamy M, Mordi DC and Prabhakar BS: MADD/DENN splice variant of the IG20 gene is necessary and sufficient for cancer cell survival. Oncogene 25: 6252-6261, 2006.

10. Schievella AR, Chen JH, Graham JR and Lin LL: MADD, a novel death domain protein that interacts with the type 1 tumor necrosis factor receptor and activates mitogen-activated protein kinase. J Biol Chem 272: 12069-12075, 1997.

11. Lee MT, Coburn GA, McClure MO and Cullen BR: Inhibition of human immunodeficiency virus type 1 replication in primary macrophages by using Tat- or CCR 5-specific small interfering RNAs expressed from a lentivirus vector. J Virol 77: 11964-11972, 2003.

12. Demirhan O, Tastemir D, Hastürk S, Kuleci S and Hanta I: Alterations in p16 and p53 genes and chromosomal findings in patients with lung cancer: fluorescence in situ hybridization and cytogenetic studies. Cancer Epidemiol 34: 472-477, 2010.

13. Lee YL, Kuo WH, Lin CW, et al: Association of genetic polymorphisms of CXCL12/SDF1 gene and its receptor, CXCR4, to the susceptibility and prognosis of non-small cell lung cancer. Lung Cancer 73: 147-152, 2011.

14. Rosell R, Moran T, Queralt C, et al: Screening for epidermal growth factor receptor mutations in lung cancer. N Engl J Med 361: 958-967, 2009.

15. Pukac L, Kanakaraj P, Humphreys R, et al: HGS-ETR1, a fully human TRAIL-receptor 1 monoclonal antibody, induces cell death in multiple tumor types in vitro and in vivo. Br J Cancer 92: 1430-1441, 2005.

16. LeBlanc HN and Ashkenazi A: Apo2L/TRAIL and its death and decoy receptors. Cell Death Differ 10: 66-75, 2003.

17. Riccioni R, Pasquini L, Mariani G, et al: TRAIL decoy receptors mediate resistance of acute myeloid leukemia cells to TRAIL. Haematologica 90: 612-624, 2005.

18. Morizot A, Mérino D, Lalaoui N, et al: Chemotherapy overcomes TRAIL-R4-mediated TRAIL resistance at the DISC level. Cell Death Differ 18: 700-711, 2011.
19. Zhang L and Fang B: Mechanisms of resistance to TRAILinduced apoptosis in cancer. Cancer Gene Ther 12: 228-237, 2005.

20. Hopkins-Donaldson S, Ziegler A, Kurtz S, et al: Silencing of death receptor and caspase- 8 expression in small cell lung carcinoma cell lines and tumors by DNA methylation. Cell Death Differ 10: 356-364, 2003.

21. Ray S, Bucur O and Almasan A: Sensitization of prostate carcinoma cells to Apo2L/TRAIL by a Bcl-2 family protein inhibitor. Apoptosis 10: 1411-1418, 2005.

22. Trauzold A, Siegmund D, Schniewind B, et al: TRAIL promotes metastasis of human pancreatic ductal adenocarcinoma. Oncogene 25: 7434-7439, 2006.

23. Cunningham SJ: Cloning and characterization of a novel cDNA isolated from human $\beta$ cells. Doctoral dissertation. The University of Texas Medical Branch, 1996.

24. Chow VT and Lee SS: DENN, a novel human gene differentially expressed in normal and neoplastic cells. DNA Seq 6: 263-273, 1996.

25. Li LC, Sheng JR, Mulherkar N, Prabhakar BS and Meriggioli MN: Regulation of apoptosis and caspase- 8 expression in neuroblastoma cells by isoforms of the IG20 gene. Cancer Res 68: 7352-7361, 2008.

26. Miyoshi J and Takai Y: Dual role of DENN/MADD (Rab3GEP) in neurotransmission and neuroprotection. Trends Mol Med 10: 476-480, 2004

27. Niwa S, Tanaka Y and Hirokawa N: KIF1Bbeta- and KIF1Amediated axonal transport of presynaptic regulator Rab3 occurs in a GTP-dependent manner through DENN/MADD. Nat Cell Biol 10: 1269-1279, 2008

28. Yamaguchi K, Tanaka M, Mizoguchi A, et al: A GDP/GTP exchange protein for the Rab3 small $\mathrm{G}$ protein family up-regulates a postdocking step of synaptic exocytosis in central synapses. Proc Natl Acad Sci USA 99: 14536-14541, 2002.

29. Del Villar K and Miller CA: Down-regulation of DENN/MADD, a TNF receptor binding protein, correlates with neuronal cell death in Alzheimer's disease brain and hippocampal neurons. Proc Natl Acad Sci USA 101: 4210-4215, 2004.

30. Kurada BR, Li LC, Mulherkar N, Subramanian M, Prasad KV and Prabhakar BS: MADD, a splice variant of IG20, is indispensable for MAPK activation and protection against apoptosis upon tumor necrosis factor-alpha treatment. J Biol Chem 284: 13533-13541, 2009.

31. Allaire PD, Marat AL, Dall'Armi C, Di Paolo G, McPherson PS and Ritter B: The connecdenn DENN domain: a GEF for Rab35 mediating cargo-specific exit from early endosomes. Mol Cell 37: 370-382, 2010

32. Prabhakar BS, Mulherkar N and Prasad KV: Role of IG20 splice variants in TRAIL resistance. Clin Cancer Res 14: 347-351, 2008.

33. Coppola T, Perret-Menoud V, Gattesco S, et al: The death domain of Rab3 guanine nucleotide exchange protein in GDP/GTP exchange activity in living cells. Biochem J 362: 273-279, 2002

34. Lim KM, Yeo WS and Chow VT: Antisense abrogation of DENN expression induces apoptosis of leukemia cells in vitro, causes tumor regression in vivo and alters the transcription of genes involved in apoptosis and the cell cycle. Int J Cancer 109: 24-37, 2004.

35. Efimova E, Martinez O, Lokshin A, Arima T and Prabhakar BS: IG20, a MADD splice variant, increases cell susceptibility to gamma-irradiation and induces soluble mediators that suppress tumor cell growth. Cancer Res 63: 8768-8776, 2003. 